\title{
Brain structural correlates of alexithymia in patients with major depressive disorder
}

\author{
Katharina Förster, MSc; Verena Enneking, MSc; Katharina Dohm, PhD; Ronny Redlich, PhD; \\ Susanne Meinert, MSc; Adina Isabel Geisler, MSc; Elisabeth Johanna Leehr, PhD; \\ Harald Kugel, PhD; Bernhard T. Baune, MD, MPH; Volker Arolt, MD; \\ Pienie Zwitserlood, PhD; Dominik Grotegerd, PhD*; Udo Dannlowski, MD, PhD*
}

\begin{abstract}
Background: Alexithymia is a risk factor for major depressive disorder (MDD) and has been associated with diminished treatment response. Neuroimaging studies have revealed structural aberrations of the anterior cingulate cortex and the fusiform gyrus in healthy controls with high levels of alexithymia. The present study tried to corroborate and extend these results to patients with MDD compared with healthy controls. Methods: We investigated the relationship between alexithymia, depression and grey matter volume in 63 patients with MDD (mean age \pm standard deviation $=42.43 \mathrm{yr} \pm 11.91$; 33 female) and 46 healthy controls $(45.35 \mathrm{yr} \pm 8.37 ; 22$ female). We assessed alexithymia using the Toronto Alexithymia Scale. We conducted an alexithymia $\times$ group analysis of covariance; we used a region-of-interest approach, including the fusiform gyrus and anterior cingulate cortex, and conducted whole brain analysis using voxelbased morphometry. Results: Our analysis revealed a significant alexithymia $\times$ group interaction in the fusiform gyrus (left, $p_{F W E}=0.031$; right, $\left.p_{F W E}=0.010\right)$. Higher alexithymia scores were associated with decreased grey matter volume in patients with MDD $\left(p_{F W E}=0.009\right)$, but with increased grey matter volume of the fusiform gyrus in healthy controls $\left(p_{F W E}=0.044\right)$. We found no significant main effects in the region-of-interest analysis. Limitations: Owing to the naturalistic nature of our study, patients with MDD and healthy controls differed significantly in their alexithymia scores. Conclusion: Our results showed the fusiform gyrus as a correlate of alexithymia. We also found differences related to alexithymia between patients with MDD and healthy controls in the fusiform gyrus. Our study encourages research related to the transition from risk to MDD in people with alexithymia.
\end{abstract}

\section{Introduction}

Major depressive disorder (MDD) is one of the most common and disabling psychiatric diseases worldwide. ${ }^{1}$ At least onethird of patients with MDD do not respond to first-line treatment, ${ }^{2}$ contributing to the burden of affective illness. ${ }^{3}$ Structural neuroimaging studies further support the importance of underlying risk factors, showing that structural brain alterations associated with MDD (such as reduced hippocampal volume) may be better explained by risk factors such as childhood maltreatment, rather than by MDD itself. ${ }^{4} \mathrm{~A}$ diagnostic and therapeutic approach based on risk factors has the advantages of differentiating subtypes in a heterogeneous category of disease, tailoring individual treatments to different subtypes of MDD, allowing prevention for people without MDD who have underlying risk factors, and transferring treatments fitted to risk factors to populations with diagnoses other than MDD.

A transdiagnostic risk factor of psychiatric disorders that is prevalent in approximately $20 \%$ of the patient population ${ }^{5}$ is a subclinical trait called alexithymia. Alexithymia is characterized by difficulty describing and identifying feelings, and is accompanied by an externally oriented emotion-processing style, ${ }^{6}$ such as avoiding emotionally deep conversations. As a result, cerebral structures that participate in emotion processing have been investigated for functional and structural alterations related to alexithymia. The anterior cingulate cortex (ACC) and the fusiform gyrus (FFG) have been repeatedly shown to be areas with functional and structural aberrations related to alexithymia, as indicated by a recent metaanalysis. ${ }^{7,8}$ The ACC monitors emotional experiences, ${ }^{9}$ which are disrupted in individuals with alexithymia. ${ }^{10}$ The fusiform

Correspondence to: U. Dannlowski, Department of Psychiatry, University of Münster, Albert-Schweitzer-Campus 1, G A9, 48149 Münster, Germany; dannlow@uni-muenster.de

*These authors contributed equally to the work and share senior authorship.

Submitted Feb. 25, 2019; Revised May 10, 2019; Accepted June 23, 2019; Published online Oct. 11, 2019

DOI: $10.1503 / j p n .190044$ 
gyrus, with its fusiform face area, is responsible for the processing of faces, ${ }^{11}$ and thus, for emotion recognition.

Although alexithymia and its neurobiological underpinnings have been thoroughly investigated in healthy individuals, $^{12}$ it remains unclear whether alexithymia is associated with specific brain structural alterations in patients with MDD and whether such alterations differ from those in healthy people. Alexithymia involves difficulties in emotion perception, and thus, relates to emotion regulation and social interaction; all 3 core processes are highly relevant to the development and course of MDD. ${ }^{13,14}$ Patients with alexithymia and MDD show an unfavourable course of disease with heightened suicide rates, ${ }^{15}$ leading to decreased response to conventional multimodal treatment approaches (antidepressant medication, cognitive behavioural therapy ${ }^{16,17}$ ). As well, alexithymia has also been discussed as a state rather than a trait predisposing people to depressive disorders. ${ }^{18,19}$ In people with depression, alexithymia has been discussed as a state reaction to mitigate negative affect. ${ }^{20,21}$ However, because few longitudinal studies are available, it remains unclear whether alexithymia in MDD is a state or a trait preceding depression. Although alexithymia and MDD are distinct and separable constructs, ${ }^{22}$ overlapping of these constructs is frequently observed..$^{15} \mathrm{~A}$ recent functional MRI study provided first insights into this entangled relationship: it identified distinct functional correlates of alexithymia in MDD and healthy controls. ${ }^{23}$ These results support the theory that alexithymia in MDD might have a different etiology or mechanism than the risk factor of alexithymia in healthy controls. A recent study investigating patients with damage to the anterior insula reported heightened alexithymia scores, indicating a link between structural brain alterations and alexithymia. ${ }^{24}$ However, structural brain alterations could be a result or a precursor of behavioural changes involved in alexithymia, such as the avoidance of emotional content. Alterations as a result of behavioural change could be caused by avoidance of emotional activity, leading to decreased activity in areas involved in emotion processing and atrophy of the involved areas. Alterations as a precursor might cause reduced activity in response to emotional stimuli and impair the identification of such stimuli as a result. Because people with MDD show structural brain alterations after relapse ${ }^{25}$ and behavioural alterations toward emotional stimuli that are associated with functional brain alterations, ${ }^{26}$ both phenomena might be present in MDD.

Patients with MDD make up a clinical population in which alexithymia is expected to be higher than in healthy controls. ${ }^{27-29}$ Previous studies comparing healthy individuals with high and low alexithymia scores showed lower grey matter volume in people with high alexithymia. ${ }^{7}$ In contrast, studies investigating healthy individuals with lower alexithymia scores have found no association or a positive association between alexithymia and grey matter volume. ${ }^{30-33}$

Our aim was to find brain structural correlates of alexithymia in lifetime MDD and brain structural correlates that were differentially associated with alexithymia in healthy controls and patients with MDD. According to the literature $^{30,32}$ people with low alexithymia scores exhibit a positive association between alexithymia and grey matter volume. Thus, we hypothesized a positive association between alexithymia and grey matter volume in FFG and ACC in the healthy controls. Because people with high alexithymia scores show a negative association between grey matter volume and alexithymia, ${ }^{30-33}$ we hypothesized that higher alexithymia would be associated with decreased grey matter volumes in patients with MDD. We examined differential structural correlates of alexithymia in depression and healthy controls in 2 different regions (the ACC and the FFG), and in an exploratory whole brain analysis.

\section{Methods}

\section{Participants and procedure}

The sample $(n=109)$ consisted of 63 patients with MDD and 46 healthy controls. Participants were recruited from the Department of Psychiatry at the University of Münster as part of the Münster Neuroimaging Cohort. Diagnoses were verified using the structured clinical interview for DSM-IV (SCID-I). ${ }^{34}$ Most of the patients with MDD were in a remitted state during the study (47 remitted, 16 acute). Remission was defined according to DSM-IV criteria: patients without an episode in the preceding 4 weeks were considered to be remitted.

Exclusion criteria for all participants were any neurologic abnormality or previous traumatic head injury, chronic medical disease, substance or alcohol abuse and dependence, or MRI contraindications. Further exclusion criteria for patients with MDD were a current or previous diagnosis of bipolar disorder or psychotic disorder, or drug or alcohol addiction, as described in previous studies. ${ }^{35}$ Of the 63 patients with MDD, 19 reported that a comorbid anxiety disorder (panic disorder, agoraphobia, or social or specific phobia) was remitted or acute during the time of the study.

Our study was approved by the local institutional ethics committee, and all participants provided written informed consent before participation. Participants were financially compensated.

\section{Behavioural measures}

\section{Alexithymia}

The Toronto Alexithymia Scale 20 (TAS-20) focuses on the cognitive dimension of alexithymia. ${ }^{6}$ The cognitive dimension centres on the cognitive processing of feelings and the ability to identify, analyze and verbalize one's feelings. The TAS-20 comprises 3 subscales: difficulty identifying feelings, difficulty describing feelings and externally oriented thinking. The TAS showed high internal consistency (Cronbach $\alpha=0.81)$ and test-retest reliability $(r=0.77)$ in a previous study. ${ }^{6}$ In the present sample, Cronbach $\alpha$ for the TAS-20 score was 0.73 . We used the TAS-20 total sum score as a measure of alexithymia. Sum scores above 61 have been associated with clinically relevant alexithymia. ${ }^{6}$ 


\section{Clinical measures}

We assessed acute symptom severity using the Hamilton Depression Rating Scale ${ }^{36}$ and the Beck Depression Inventory. ${ }^{37}$ We assessed cumulative illness severity using number of lifetime hospitalizations. ${ }^{35,38}$ We assessed psychopharmacological medication use in patients with MDD to compute a medication load index, as described in previous studies. ${ }^{39}$

\section{Structural MRI}

\section{Data acquisition and preprocessing}

We acquired $T_{1}$-weighted high-resolution anatomic images using a 3 T MRI scanner (Gyroscan Intera 3T; Philips Medical Systems): 3D fast gradient echo sequence (turbo field echo); repetition time $7.4 \mathrm{~ms}$, echo time $3.4 \mathrm{~ms}$, flip angle $98^{\circ}$, from 2 signal averages, inversion prepulse every $814.5 \mathrm{~ms}$, field of view $256 \mathrm{~mm} \times 204 \mathrm{~mm} \times 160 \mathrm{~mm}$, phase encoding in anterior-posterior and right-left directions, reconstructed to cubic voxels of $0.5 \mathrm{~mm} \times 0.5 \mathrm{~mm} \times 0.5 \mathrm{~mm}$. We used the computational anatomy toolbox (cat12-toolbox v1184, see http:/ / dbm.neuro.uni-jena.de/cat/) for preprocessing, with default parameters. Briefly, images were bias-corrected, tissueclassified and normalized, and the resulting grey matter segments were smoothed ( $8 \mathrm{~mm}$ full width at half maximum). We carefully checked the quality of the segmentation results for outliers and artifacts.

\section{Statistical analyses}

We analyzed the data using statistical parametric mapping (SPM12, Wellcome Trust Centre for Neuroimaging, London, http://www.fil.ion.ucl.ac.uk/spm/). We defined the ACC and FFG regions of interest (ROIs) using the AAL-Atlas ${ }^{40}$ implemented in the Wake Forest University Pickatlas (http:/ / fmri.wfubmc.edu/software/PickAtlas). We conducted an alexithymia (TAS-20) $\times$ group (MDD, healthy controls) analysis of covariance using age, sex and total intracranial volume as nuisance regressors in all analyses (model 1). We calculated a priori-defined $t$ contrasts according to our hypotheses.

To investigate whether the results were stable and independent of former course of illness, antidepressant treatment and remission status, we conducted a multiple regression analysis in our ROIs using alexithymia as a regressor of interest (model 2) in the MDD subgroup, and lifetime number of hospitalizations, medication index and remission (dummy-coded according to SCID-I diagnosis: remission $=1$; no remission $=0$ ) as covariates of no interest. We then repeated this analysis controlling for comorbid anxiety disorders (dummy-coded according to SCID-I diagnosis: comorbid anxiety disorder $=1$; no anxiety disorder $=0$ ) and depression severity measured using the Hamilton Depression Rating Scale instead of remission status.

In addition to the ROI analysis of the ACC and the FFG, we conducted a group $\times$ alexithymia analysis of covariance controlling for age, sex and total intracranial volume as a whole brain analysis (model 1) to explore the potential effects on grey matter volume of other brain regions. To determine the statistical significance of putative clusters, we used the nonparametric approach of threshold-free cluster enhance- ment (TFCE), as implemented in the TFCE toolbox (http:/ / dbm.neuro.uni-jena.de/tfce; version 140). We applied rigorous family-wise error (FWE) correction at a threshold of $p_{\mathrm{FWE}}<0.05$ obtained by 5000 permutations per test. ${ }^{41}$

We conducted subsequent analysis in SPSS 25 (SPSS Inc.) using the extracted values of significant clusters (Appendix 1, available at jpn.ca/190044-a1; supplementary analyses 1 and 2).

\section{Results}

\section{Sample characteristics}

An overview of sample characteristics is given in Table 1 (lifetime comorbidities of the MDD sample) and Table 2 (sociodemographic and clinical characteristics of the both study

\begin{tabular}{lc} 
Table 1: Major depressive disorder sample, comorbidities* \\
\hline Lifetime comorbidity & No. \\
\hline Substance abuse & 7 \\
Cannabis & 3 \\
Sedatives & 1 \\
Alcohol & 3 \\
Eating disorder & 2 \\
Bulimia nervosa & 1 \\
Binge-eating disorder & 1 \\
Obsessive-compulsive disorder & 2 \\
Posttraumatic stress disorder & 3 \\
Anxiety disorder & 19 \\
Social phobia & 7 \\
Specific phobia & 2 \\
Panic disorder with/without agoraphobia & 6 \\
Agoraphobia without panic disorder & 4
\end{tabular}

*According to DSM-IV diagnosis. Comorbidities could be remitted, partly remitted or acute.

Table 2: Sociodemographic and clinical characteristics*

\begin{tabular}{|c|c|c|c|}
\hline Characteristic & $\begin{array}{l}\text { Major } \\
\text { depressive } \\
\text { disorder } \\
(n=63)\end{array}$ & $\begin{array}{l}\text { Healthy } \\
\text { controls } \\
(n=46)\end{array}$ & $p$ value \\
\hline \multicolumn{4}{|c|}{ Sociodemographic characteristics } \\
\hline Age, yr & $42.43 \pm 11.91$ & $45.35 \pm 8.37$ & 0.16 \\
\hline Female, no. & 33 & 22 & $0.64 \ddagger$ \\
\hline \multicolumn{4}{|l|}{ Questionnaire scores } \\
\hline $\begin{array}{l}\text { Toronto Alexithymia } \\
\text { Scale, } 20 \text {-item form }\end{array}$ & $53.16 \pm 10.41$ & $39.14 \pm 10.74$ & $<0.001$ \\
\hline $\begin{array}{l}\text { Beck Depression } \\
\text { Inventory }\end{array}$ & $17.66 \pm 12.21$ & $2.89 \pm 3.12$ & $<0.001$ \\
\hline $\begin{array}{l}\text { Hamilton Depression } \\
\text { Rating Scale }\end{array}$ & $11.33 \pm 7.88$ & $0.91 \pm 1.35$ & $<0.001$ \\
\hline \multicolumn{4}{|l|}{ Clinical characteristics } \\
\hline Inpatient treatments, no & $2.67 \pm 1.93$ & - & - \\
\hline Medication load index & $1.33 \pm 1.64$ & - & - \\
\hline \multicolumn{4}{|c|}{$\begin{array}{l}{ }^{\star} \text { Findings are shown as mean } \pm \text { standard deviation unless otherwise indicated. } \\
\text { †Obtained using the unpaired } 2 \text {-tailed } t \text { test, except where noted. } \\
\text { †Obtained using the } \chi^{2} \text { test. }\end{array}$} \\
\hline
\end{tabular}


samples). Patients with acute MDD showed significantly higher alexithymia scores than patients with MDD in remission (remitted MDD, mean \pm standard deviation $=51.53 \pm$ 9.98; acute MDD, $57.94 \pm 10.49 ; t_{61}=-2.190, p=0.032$ ). Patients with MDD in remission exhibited significantly higher alexithymia scores than healthy controls $\left(t_{91}=-5.770, p<\right.$ 0.001). Alexithymia was associated with intensity of selfreported depressive symptoms (Beck Depression Inventory) across the whole sample $(r=0.552 ; p<0.001)$ as well as in the $\operatorname{MDD}(r=0.391 ; p=0.001)$ and healthy controls $(r=0.297 ; p=$ $0.024)$ samples alone.

Alexithymia and intensity of suicidal thoughts (measured using the Beck Depression Inventory) were correlated in patients with $\operatorname{MDD}\left(r_{s}=0.347 ; p=0.003\right)$. In patients with acute MDD, intensity of suicidal thoughts and TAS-20 score were significantly associated. Higher alexithymia was associated with higher intensity of suicidal thoughts $\left(r_{s}=0.471 ; p=\right.$ 0.033). In patients with MDD in remission, the relationship of alexithymia and suicidal thoughts showed only a trend toward significance $\left(r_{s}=0.214 ; p=0.077\right)$.

\section{Main effect of alexithymia on grey matter volume (model 1)}

The ROI analyses of ACC and FFG volume showed no main effect of alexithymia on grey matter volume. The exploratory whole brain analyses revealed clusters in 4 separate regions. Higher alexithymia was associated with increased grey matter volume in both groups (Table 1).

\section{Alexithymia $\times$ group interaction on grey matter volume (model 1)}

We found a significant interaction of alexithymia $\times$ group in the bilateral FFG (right: $k=932, t_{102}=3.58, x=28, y=-44, z=$ $-16, p_{\mathrm{FWE}}=0.010$; left: $k=116, t_{102}=3.49, x=-27, y=-57, z=$ $-15, p_{\mathrm{FWE}}=0.031$; Fig. 1 and Fig. 2). Higher TAS-20 scores were associated with increased FFG volume in healthy controls (right: $k=30, t_{102}=3.34, x=22, y=-45, z=-14, p_{\mathrm{FWE}}=$ $0.044, r=0.328)$, but with decreased FFG volume in patients with MDD (left: $k=320, t_{102}=-2.90, x=-27, y=-57, z=-15$, $\left.p_{\mathrm{FWE}}=0.009, r=-0.390\right)$. We found no significant interaction effects in the ROI analysis of the ACC.

Analyzing differential effects of both groups in an exploratory whole brain analysis revealed that higher alexithymia was associated with increased grey matter in 4 regions previously reported to be associated with alexithymia in healthy controls (e.g., the fusiform gyrus; Table 3). In patients with MDD, higher alexithymia was associated only with higher volume in the left insula (Table 3).

Negative association of alexithymia with grey matter volume in the MDD sample (model 2)

In a separate analysis of the MDD group - adjusting for the number of hospitalizations, remission of depression as a binary variable and current medication - the cluster in the FFG remained significant $\left(k=313, t_{55}=3.92, x=-27, y=-58\right.$, $\left.z=-16, p_{\mathrm{FWE}}=0.020, r=-0.490\right)$. Alexithymia was still signifi-

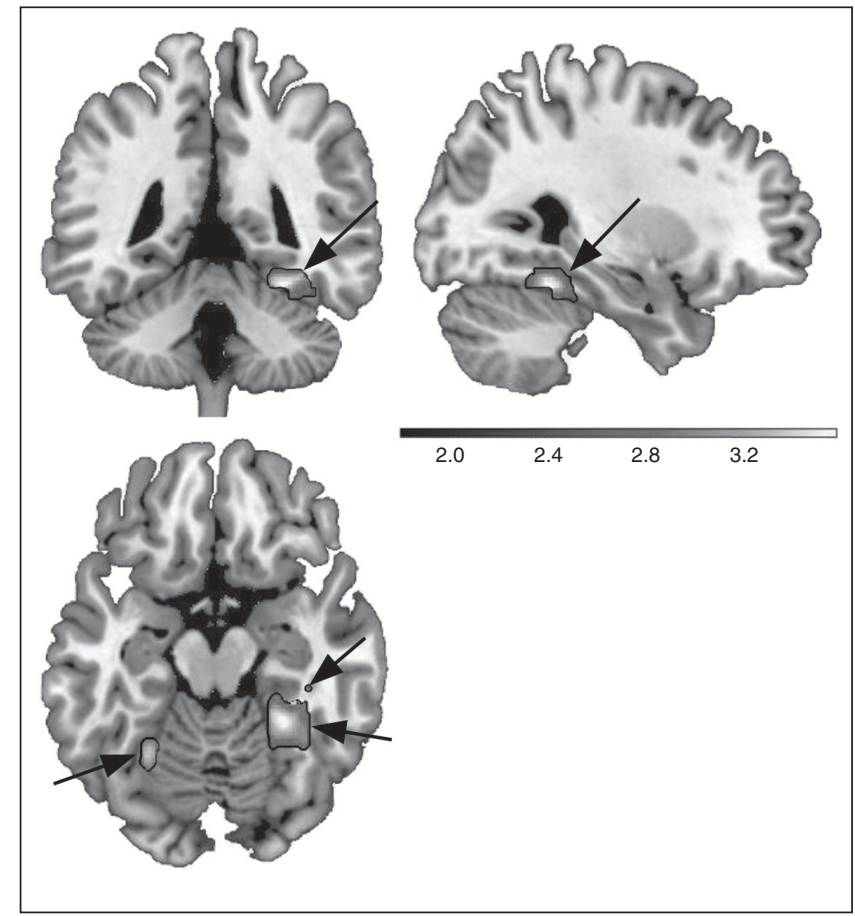

Fig. 1: Interaction of diagnosis (patients with major depressive disorder, healthy controls) with alexithymia on the structure of the fusiform gyrus. Coronal, sagittal and axial views $(x=28, y=-44, z=$ $-16)$ of the region-of-interest analysis in the fusiform gyrus. The $t$ contrast in the analysis of covariance model revealed a positive association between alexithymia and grey matter volume in controls, but a negative relationship in patients with major depressive disorder. Shaded bar represents the $t$ value of the cluster.

cantly associated with fusiform gyrus volume when severity of depression (Hamilton Depression Rating Scale) and comorbid anxiety were used as additional nuisance regressors instead of remission status $\left(k=93, t_{54}=3.57, x=-30, y=-58\right.$, $\left.z=-18, p_{\mathrm{FWE}}=0.038, r=-0.483\right)$.

\section{Nonlinear regression analysis}

Considering the distribution of the present alexithymia scores (Fig. 2), we investigated whether a quadratic function was a better representation of the data (Appendix 1, supplementary analysis 2).

\section{Main effect of group on grey matter volume (model 1)}

We found no significant main effect that survived rigorous $\alpha$ correction for the factor group, either in the ROI analyses of the ACC and the FFG, or at the whole brain level.

\section{Discussion}

The present work investigated the relationship between alexithymia and structural brain alterations in patients with depression. We addressed the question of whether alexithymia might be a state phenomenon in patients with MDD. To our 


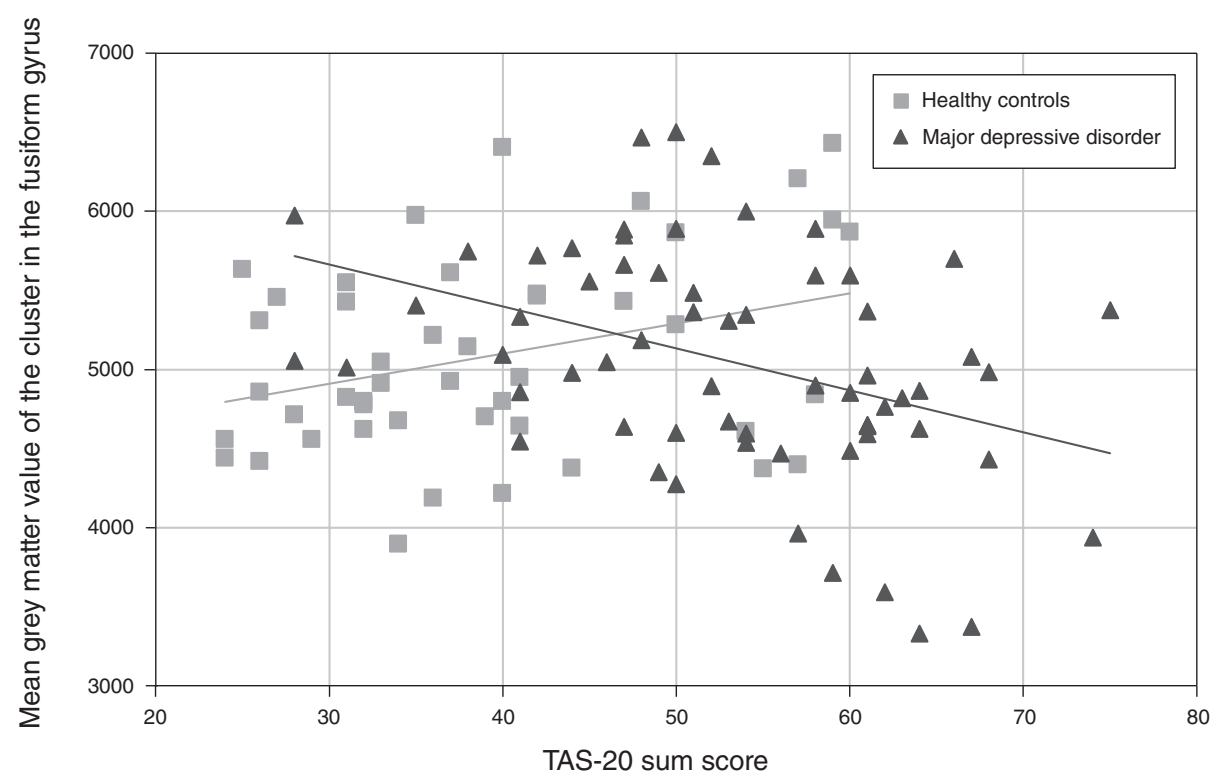

Fig. 2: The Toronto Alexithymia Scale 20 (TAS-20) correlated with grey matter volume (mean grey matter value of the cluster at $x=28, y=-44, z=-16$ ) of the bilateral fusiform gyrus separated by group. Continuous lines represent regression slopes separated by group.

knowledge, we provided the first evidence that patients with MDD show a different relationship between alexithymia and brain structure than healthy controls. Patients and controls both showed an association of FFG volume with alexithymia. In the FFG, higher alexithymia scores were associated with increased grey matter volume in healthy controls, but with decreased volume in patients with MDD. In the patient group, the results were stable even when former course of illness, psychopharmacological treatment and remission status were entered as covariates of no interest. The persistent stability of results indicated that the course and severity of depression, as well as medication, did not contribute to the differential expression of grey matter volume in the patient group.

The FFG is a brain structure responsible for recognizing faces, bodies and body parts. ${ }^{42,43}$ Structural damage to the FFG leads to prosopagnosia, the inability to identify faces. ${ }^{44}$ The identification of individuals is important for maintaining functional social relationships, ${ }^{45,46}$ a factor that has been associated with better recovery from depression and resilience to psychopathology. ${ }^{47}$ Further, face recognition is an important factor that contributes to emotion recognition, which is impaired in people with alexithymia. ${ }^{48}$ Recent research points to the avoidance of emotional facial expressions in people with high levels of alexithymia compared to those with low alexithymia, ${ }^{49}$ indicating difficulties with the identification or interpretation of facial affect. This result fits well with our finding of lower FFG volume in association with high alexithymia scores in patients with MDD. Facial affect recognition might be a promising target for intervention in people with high alexithymia. Moreover, functional changes in the FFG
Table 3: Main positive effects of alexithymia on brain structure*

\begin{tabular}{|c|c|c|c|c|}
\hline Region & k & $t$ & $p_{\mathrm{FWE}}$ & $\begin{array}{c}\text { MNI } \\
\text { coordinates, } \\
x, y, z\end{array}$ \\
\hline \multicolumn{5}{|c|}{ Main effect of alexithymia, independent of group } \\
\hline Left insula & 636 & 3.17 & 0.024 & $-34,-8,10$ \\
\hline Right temporal inferior & 433 & 3.27 & 0.026 & $56,-3,-32$ \\
\hline Left temporal inferior & 303 & 3.31 & 0.033 & $-56,-3,-34$ \\
\hline Right thalamus & 235 & 2.99 & 0.044 & $10,-24,10$ \\
\hline \multicolumn{5}{|c|}{ Effect of alexithymia in patients with major depressive disorder } \\
\hline Left insula & 45 & 4.14 & 0.046 & $-45,3,9$ \\
\hline \multicolumn{5}{|c|}{ Effect of alexithymia in healthy controls } \\
\hline $\begin{array}{l}\text { Right hippocampus, } \\
\text { thalamus, fusiform gyrus }\end{array}$ & 1846 & 4.67 & 0.010 & $22,-33,6$ \\
\hline Right medial cingulum & 205 & 3.89 & 0.034 & $9,-22,38$ \\
\hline $\begin{array}{l}\text { Left and right posterior } \\
\text { cingulum }\end{array}$ & 142 & 3.55 & 0.039 & $6,0,15$ \\
\hline Right fusiform gyrus & 22 & 3.13 & 0.049 & $36,-46,-12$ \\
\hline \multicolumn{5}{|c|}{$\begin{array}{l}\text { FWE = family-wise error; MNI = Montreal Neurological Institute. } \\
{ }^{*} \text { Model was an analysis of covariance, with group as a factor and alexithymia sum } \\
\text { scores as covariates. Age, sex and total intracranial volume were included as } \\
\text { nuisance regressors. Values represent the main effect of alexithymia on brain } \\
\text { structure across both groups and in each group in an exploratory whole brain analysis. } \\
\text { For brevity, the main effects of alexithymia independent of group are reported only } \\
\text { above a cluster size of } 200 \text { voxels. }\end{array}$} \\
\hline
\end{tabular}

have been associated with alexithymia. ${ }^{50,51}$ Our results support previous studies showing that the FFG is a neuronal correlate of alexithymia. ${ }^{50,52,53}$ Our analysis revealed a positive relationship between FFG volume and alexithymia in an ROI approach. Strengthening this effect, we found a significant 
main effect of alexithymia on grey matter volume only in healthy controls. The cluster contained approximately 90 voxels of FFG volume (5\% of the cluster). In contrast to previous studies, higher volume in the FFG was associated with higher alexithymia. Studies showing a positive association between alexithymia and brain volume ${ }^{30}$ looked mainly at correlation in a group of participants with lower alexithymia scores, comparable to our group of healthy controls.

Consistent with our hypothesis, we showed that alexithymia in MDD was associated with decreased volume in the FFG. This could be explained by different mechanisms. One possibility is the neurotoxicity hypothesis: that within depression, changes in the activation of the hypothalamic-pituitaryadrenal axis lead to prolonged secretion of glucocorticoids, which increase ordinary attrition of brain structures. ${ }^{54}$ In contrast, the vulnerability hypothesis states that the structural brain alterations precede the onset of depression and are a result of risk factors such as childhood maltreatment or alexithymia. ${ }^{4}$ Hence, lower volume in the hippocampus might be a result of the transdiagnostic risk factor childhood maltreatment rather than depression itself. In contrast to the main effect of maltreatment, which manifested similarly in healthy controls and people with MDD, alexithymia showed distinct associations with FFG volume that was dependent on diagnostic status. Major depressive disorder might lead to a different expression of alexithymia and hence a different expression of neurobiological alterations associated with alexithymia. However, a coping mechanism in healthy controls might also lead to different expression of FFG volume. People with alexithymia might rely on face recognition to ease the identification of emotions, which could explain increased volumes in this area and compensate for the alexithymia. Thus, we speculate that this correlate is associated with protection rather than vulnerability to depression. However, we only can hypothesize about the relationship between structural correlates and brain function. As well, it remains unknown how the the onset of depression affects the relationship between FFG volume and alexithymia. Lower FFG volume might still precede depression in people with alexithymia if compensatory mechanisms lead to increased stress over the long term. Surprisingly, we also found the negative association between alexithymia and FFG volume when we limited the analysis to patients with MDD in remission who showed significantly lower alexithymia scores than patients with acute MDD. If alexithymia were a state in patients with MDD, we would assume that the structural correlates of alexithymia would not be present in patients with MDD in remission. One explanation for this finding could be the fact that remitted patients still showed higher alexithymia scores than healthy controls, suggesting a trait characteristic of alexithymia. Nonetheless, in patients with MDD, it remains unknown whether the negative relationship between alexithymia and FFG volume is already present before the onset of MDD, or whether it is a result of MDD. In addition, we revealed a relationship between higher alexithymia and lower FFG volume in the acute MDD group that showed a trend toward significance. Unfortunately, we had only 16 patients with acute MDD in our study. Considering the high correlation between the mean cluster value and alexithymia in the acute MDD group, it is possible that the effect would have been significant in a bigger sample of patients with acute MDD. A stable negative relationship between alexithymia and FFG volume in acute and remitted patients compared to a positive relationship in healthy controls would indicate that the relationship of alexithymia with grey matter volume is dependent on a lifetime diagnosis of MDD but not dependent on acute depression status. It remains difficult to draw conclusions about the state versus trait debate with respect to alexithymia. A larger sample is needed to statistically fortify this hypothesis. Hence, lower FFG volume could be a precursor of MDD, or a result of MDD, or both. Two questions following from our interpretation should be further investigated in well-powered longitudinal studies using healthy controls with high alexithymia as a risk population for MDD: (1) Do healthy individuals with high alexithymia scores already show a negative association between alexithymia and FFG volume; or (2) Does the relationship between alexithymia and FFG volume reverse after the onset of MDD?

Our results contribute to the debate about treatment approaches that target transdiagnostic processes such as emotion perception in depression. Applying neurobiological correlates of depression, research can further resolve the heterogeneity of the MDD diagnosis and contribute to a tailored approach in psychiatry and psychotherapy. Because patients with MDD and alexithymia are prone to a worse course of disease and higher suicidality, MDD-specific treatment of these patients needs further adjustment. The FFG and its functional role could be a promising target for developing prevention and intervention methods for people with alexithymia. Functional neuroimaging studies show that the FFG is involved in the recognition of faces ${ }^{11}$ and emotion recognition, ${ }^{55}$ which is compromised in patients with MDD and in healthy people with alexithymia. A targeted intervention could train facial affect recognition to ease the identification and description of emotion, which might lead to lower avoidance of emotional content.

Further research should evaluate the effect of psychotherapy and/or prevention that focuses on facial emotion recognition and regulation on the FFG. Considering the often-reported negative interpretation bias in facial affect recognition in patients with $\mathrm{MDD}^{56}$ an intervention that modulates affect recognition itself should also be considered for further investigation.

In contrast to our findings for the FFG, we observed no changes to the ACC in either group. This finding was in line with a recent meta-analysis that also reported no structural alterations in the ACC associated with alexithymia. ${ }^{12}$ One reason might be the heterogeneity of reported volumetric changes in the ACC. Structural changes of other parts of the cingulate cortex, including the posterior and medial cingulate cortex, have been reported previously and were replicated in the present work. ${ }^{57,58}$ As well, we identified the insula and the inferior temporal lobe as structural brain correlates of alexithymia. The insula was identified as a structural brain correlate in a recent meta-analysis, further strengthening the relationship between structural alterations in the insula and behavioural changes similar to 
alexithymia. ${ }^{12}$ The inferior temporal lobe has been identified as a structural correlate of alexithymia in a general population sample, ${ }^{52}$ a finding we replicated in this study. In contrast to previous results, our results indicate a positive relationship between brain volumes in the respective brain structures and the intensity of alexithymia.

\section{Limitations}

This work had several limitations. Because alexithymia is highly prevalent in clinical populations, especially in patients with MDD, it is very probable that our MDD group reached higher scores of alexithymia than our control group. Because of the naturalistic nature of our sample, we did not screen for alexithymia beforehand. Future studies should include healthy controls with increased alexithymia scores to further investigate the relationship between alexithymia and grey matter volume, as well as the transition from high risk status to the onset of depression.

The interaction we found was not present when we modelled alexithymia as a quadratic function. However, based on our current sample, we cannot conclude that the relationship between alexithymia and grey matter volume is best represented by a quadratic function. Our sample consisted of MDD patients and healthy controls who differed significantly in mean alexithymia score; thus, drawing conclusions from the relationship between squared alexithymia and FFG grey matter volume would mean drawing conclusions from a spurious correlation.

We could not find a group difference in grey matter volume between patients with MDD and healthy controls. This might have been because more than two-thirds of our MDD sample were in a remitted state during the study. Although few studies have reported brain structural differences between patients with MDD in remission and healthy controls, ${ }^{59}$ we failed to identify those in our sample. However, we conducted this investigation in a small sample of patients with a mixed clinical course of disease and different mood states. Previous studies that analyzed structural differences between patients with MDD in remission and healthy controls were well designed to find this effect. For example, one study investigated unmedicated participants. ${ }^{59}$ In another study addressing structural brain alterations in patients with MDD, Schmaal and colleagues used a sample of 2148 patients, resulting in rather small effect sizes. ${ }^{60}$ Our sample size might have been too low to detect small effect sizes, especially when applying rigorous $\alpha$ correction procedures.

\section{Conclusion}

Our study investigated how healthy controls and patients with MDD differed in brain structural alterations associated with alexithymia. Our aim was to find brain structural correlates of alexithymia in MDD and brain structural correlates that were differentially associated with alexithymia in healthy controls and patients with MDD. We observed that FFG volume was differentially associated with alexithymia in healthy controls and patients with MDD. We hypothesize that a different mechanism contributes to the structural brain alterations associated with alexithymia in patients with MDD compared with healthy controls.

Affiliations: From the Department of Psychiatry, University of Münster, Münster, Germany (Förster, Enneking, Dohm, Redlich, Meinert, Geisler, Leehr, Baune, Arolt, Grotegerd, Dannlowski); the Institute of Clinical Radiology, University of Münster, Münster, Germany (Kugel); the Department of Psychiatry, University of Melbourne, Parkville, Australia (Baune); the Department of Psychology, University of Münster, Münster, Germany (Zwitserlood); and the Florey Institute for Neuroscience and Mental Health, University of Melbourne, Parkville, Australia (Baune).

Funding: This work was funded by the German Research Foundation (DFG, grant FOR2107 DA1151/5-1 and DA1151/5-2 to U. Dannlowski; SFB-TRR58, Projects C09 and Z02 to U. Dannlowski) and the Interdisciplinary Centre for Clinical Research (IZKF) of the medical faculty of Münster (grant Dan3/012/17 to U. Dannlowski).

Competing interests: $\mathrm{H}$. Kugel received consultation fees from MR:comp GmbH, Testing Services for MR Safety, unrelated to the present work. V. Arolt is a member of the advisory board of, or has given presentations on behalf of, the following companies: AstraZeneca, Janssen-Organon, Lilly, Lundbeck, Servier, Pfizer, Otsuka, and Trommsdorff. K. Förster, V. Enneking, K. Dohm, R. Redlich, S. Meinert, A. Geisler, E. Leehr, B. Baune, P. Zwitserlood, D. Grotegerd and U. Dannlowski declare no competing interests.

Contributors: K. Förster, D. Grotegerd and U. Dannlowski designed the study. K. Förster, V. Enneking, K. Dohm, R. Redlich, S. Meinert, A.Geisler, E. Leehr, H. Kugel and D. Grotegerd acquired the data, which K. Förster, V. Enneking, R. Redlich, B. Baune, V. Arolt, P. Zwitserlood, D. Grotegerd and U. Dannlowski analyzed. K. Förster and U. Dannlowski wrote the article, which all authors reviewed. All authors approved the final version to be published and can certify that no other individuals not listed as authors have made substantial contributions to the paper.

\section{References}

1. Wittchen H-U, Jacobi F. Size and burden of mental disorders in Europe-a critical review and appraisal of 27 studies. Eur Neuropsychopharmacol 2005;15:357-76.

2. Warden D, Rush AJ, Trivedi $\mathrm{MH}$, et al. The STAR*D Project results: a comprehensive review of findings. Curr Psychiatry Rep 2007;9:449-59.

3. Gibson TB, Jing Y, Smith Carls G, et al. Cost burden of treatment resistance in patients with depression. Am J Manag Care 2010;16: 370-7.

4. Opel N, Redlich R, Zwanzger P, et al. Hippocampal atrophy in major depression: a function of childhood maltreatment rather than diagnosis? Neuropsychopharmacology 2014;39:2723-31.

5. Luminet O, Bagby RM, Taylor GJ. An evaluation of the absolute and relative stability of alexithymia in patients with major depression. Psychother Psychosom 2001;70:254-60.

6. Bagby RM, Parker JDA, Taylor GJ. The twenty-item Toronto Alexithymia scale-I. Item selection and cross-validation of the factor structure. J Psychosom Res 1994;38:23-32.

7. Xu P, Opmeer EM, Van Tol M, et al. Structure of the alexithymic brain: a parametric coordinate-based meta-analysis. Neurosci Biobehav Rev 2018;87:50-5.

8. van der Velde J, Servaas MN, Goerlich KS, et al. Neural correlates of alexithymia: a meta-analysis of emotion processing studies. Neurosci Biobehav Rev 2013;37:1774-85.

9. Medford N, Critchley HD. Conjoint activity of anterior insular and anterior cingulate cortex: awareness and response. Brain Struct Funct 2010;214:535-49.

10. Lane RD, Ahern GL, Schwartz GE, et al. Is alexithymia the emotional equivalent of blindsight? Biol Psychiatry 1997;42:834-44. 
11. Mccarthy G, Puce A, Gore JC, et al. Face-specific processing in the human fusiforrn gyrus. J Cogn Neurosci 1997;9:605-10.

12. Xu P, Opmeer EM, van Tol M-J, et al. Structure of the alexithymic brain: a parametric coordinate-based meta-analysis. Neurosci Biobehav Rev 2018;87:50-5.

13. Shiroma PR, Thuras P, Johns B, et al. Emotion recognition processing as early predictor of response to 8-week citalopram treatment in late-life depression. Int J Geriatr Psychiatry 2014;29:1132-9.

14. Bourke C, Douglas K, Porter R. Processing of facial emotion expression in major depression: a review. Aust N Z J Psychiatry 2010;44:681-96.

15. Hintikka J, Honkalampi K, Koivumaa-Honkanen H, et al. Alexithymia and suicidal ideation: a 12-month follow-up study in a general population. Compr Psychiatry 2004;45:340-5.

16. Simson U, Martin K, Schäfer R, et al. Changes in alexithymia and emotional awareness during psychotherapeutic inpatient treatment. Psychother Psychosom Med Psychol 2006;56:376-82.

17. Günther V, Rufer M, Kersting A, et al. Predicting symptoms in major depression after inpatient treatment: the role of alexithymia. Nord J Psychiatry 2016;70:392-8.

18. Haviland MG, Shaw DG, Cummings MA, et al. Alexithymia: subscales and relationship to depression. Psychother Psychosom 1988;50:164-70.

19. Wise TN, Mann LS, Mitchell JD, et al. Secondary alexithymia: an empirical validation. Compr Psychiatry 1990;31:284-8.

20. Wise TN, Jani NN, Kass E, et al. Alexithymia: relationship to severity of medical illness and depression. Psychother Psychosom 1988;50:68-71

21. Parker JDA, Bagby RM, Taylor GJ. Alexithymia and depression: distinct or overlapping constructs? Compr Psychiatry 1991;32:387-94.

22. Marchesi C, Brusamonti E, Maggini C. Are alexithymia, depression, and anxiety distinct constructs in affective disorders? I Psychosom 2000;49:43-9

23. Ho NSP, Wong MMC, Lee TMC. Neural connectivity of alexithymia: specific association with major depressive disorder. J Affect Disord 2016;193:362-72.

24. Hogeveen J, Bird G, Chau A, et al. Acquired alexithymia following damage to the anterior insula. Neuropsychologia 2016;82:142-8.

25. Zaremba D, Dohm K, Redlich R, et al. Association of brain cortical changes with relapse in patients with major depressive disorder. JAMA Psychiatry 2018;75:484

26. Cusi AM, Nazarov A, Holshausen K, et al. Systematic review of the neural basis of social cognition in patients with mood disorders. J Psychiatry Neurosci 2012;37:154-69.

27. Bankier B, Aigner M, Bach M. Alexithymia in DSM-IV disorder: comparative evaluation of somatoform disorder, panic disorder, obsessive-compulsive disorder, and depression. Psychosomatics 2001; 42:235-40.

28. Duddu V, Isaac M, Chaturvedi S. Alexithymia in somatoform and depressive disorders. I Psychosom 2003;54:435-8.

29. Leweke F, Leichsenring F, Kruse J, et al. Is alexithymia associated with specific mental disorders? Psychopathology 2012;45:22-8

30. Gündel H, López-Sala A, Ceballos-Baumann AO, et al. Alexithymia correlates with the size of the right anterior cingulate. Psychosom Med 2004;66:132-40.

31. Heinzel A, Minnerop M, Schäfer R, et al. Alexithymia in healthy young men: a voxel-based morphometric study. J Affect Disord 2012; 136:1252-6

32. Aust $S$, Stasch J, Jentschke $S$, et al. Differential effects of early life stress on hippocampus and amygdala volume as a function of emotional abilities. Hippocampus 2014;24:1094-101.

33. Zhang X, Salmeron BJ, Ross TJ, et al. Factors underlying prefrontal and insula structural alterations in smokers. Neuroimage 2010; 54:42-8.

34. Wittchen H-U, Wunderlich U, Gruschwitz S, et al. SKID-I. Strukturiertes Klinisches Interview für DSM-IV. Göttingen, Germany: Hogrefe; 1997.

35. Zaremba D, Enneking V, Meinert S, et al. Effects of cumulative illness severity on hippocampal gray matter volume in major depression: a voxel-based morphometry study. Psychol Med 2018;48:2391-8.
36. Hamilton M. A rating scale for depression. I Neurol Neurosurg Psychiatry 1960;23:56-62.

37. Beck AT, Steer RA, Brown GK. Beck depression inventory-II. San Antonio (TX): Psychological Corporation; 1996.

38. Zaremba D, Dohm K, Redlich R, et al. Association of brain cortical changes with relapse in patients with major depressive disorder. JAMA Psychiatry 2018;75:484-92.

39. Redlich R, Almeida JJR, Grotegerd D, et al. Brain morphometric biomarkers distinguishing unipolar and bipolar depression. JAMA Psychiatry 2014;71:1222-30.

40. Tzourio-Mazoyer N, Landeau B, Papathanassiou D, et al. Automated anatomical labeling of activations in SPM using a macroscopic anatomical parcellation of the MNI MRI single-subject brain. Neuroimage 2002;15:273-89.

41. Smith SM, Nichols TE. Threshold-free cluster enhancement: addressing problems of smoothing, threshold dependence and localisation in cluster inference. Neuroimage 2009;44:83-98.

42. McCarthy G, Puce A, Gore JC, et al. Face-specific processing in the human fusiforrn gyrus. J Cogn Neurosci 1997;9:605-10.

43. Weiner KS, Grill-Spector K. The improbable simplicity of the fusiform face area. Trends Cogn Sci 2012;16:251-4.

44. Rypma B, Fischer H, Rieckmann A, et al. Dopamine D1 binding potential predicts fusiform BOLD activity during face-recognition performance. J Neurosci. 2015;35:14702-7.

45. Yardley L, McDermott L, Pisarski S, et al. Psychosocial consequences of developmental prosopagnosia: a problem of recognition. J Psychosom 2008;65:445-51.

46. Garrido-Vásquez P, Jessen S, Kotz SA. Perception of emotion in psychiatric disorders: on the possible role of task, dynamics, and multimodality. Soc Neurosci 2011;6:515-36.

47. George LK, Blazer DG, Hughes DC, et al. Social support and the outcome of major depression. Br J Psychiatry 1989;154:478-85.

48. Lane RD, Sechrest L, Riedel R, et al. Pervasive emotion recognition deficit common to alexithymia and the repressive coping style. Psychosom Med 2000;62:492-501.

49. Fujiwara E. Looking at the eyes interferes with facial emotion recognition in alexithymia. J Abnorm Psychol 2018;127:571-7.

50. Eichmann M, Kugel H, Suslow T. Difficulty identifying feelings and automatic activation in the fusiform gyrus in response to facial emotion. Percept Mot Skills 2008;107:915-22.

51. Duan X, Dai Q, Gong Q, et al. Neural mechanism of unconscious perception of surprised facial expression. Neuroimage 2010;52:401-7.

52. Grabe HJ, Wittfeld K, Hegenscheid K, et al. Alexithymia and brain gray matter volumes in a general population sample. Hum Brain Mapp 2014;35:5932-45.

53. Reker M, Ohrmann P, Rauch AV, et al. Individual differences in alexithymia and brain response to masked emotion faces. Cortex 2010;46:658-67.

54. Sapolsky RM. The possibility of neurotoxicity in the hippocampus in major depression: a primer on neuron death. Biol Psychiatry 2000;48:755-65.

55. Stuhrmann A, Suslow T, Dannlowski U. Facial emotion processing in major depression: a systematic review of neuroimaging findings. Biol Mood Anxiety Disord 2011;1:10.

56. Weightman MJ, Air TM, Baune BT. A review of the role of social cognition in major depressive disorder. Front Psychiatry 2014;5:179.

57. Paradiso S, Vaidya JG, McCormick LM, et al. Aging and alexithymia: association with reduced right rostral cingulate volume. Am J Geriatr Psychiatry 2008;16:760-9.

58. D'Agata F, Caroppo P, Amianto F, et al. Brain correlates of alexithymia in eating disorders: a voxel-based morphometry study. Psychiatry Clin Neurosci 2015;69:708-16.

59. Salvadore G, Nugent AC, Lemaitre H, et al. Prefrontal cortical abnormalities in currently depressed versus currently remitted patients with major depressive disorder. Neuroimage 2011;54:2643-51.

60. Schmaal L, Hibar DP, Sämann PG, et al. Cortical abnormalities in adults and adolescents with major depression based on brain scans from 20 cohorts worldwide in the ENIGMA Major Depressive Disorder Working Group. Mol Psychiatry 2017;22:900-9. 